\title{
Template-Assisted Hydrothermal Growth of Aligned Zinc Oxide Nanowires for Piezoelectric Energy Harvesting Applications
}

\author{
Canlin Ou, ${ }^{\dagger}$ Pedro E. Sanchez-Jimenez, ${ }^{\dagger, \dagger}$ Anuja Datta, ${ }^{\dagger}$ Francesca L. Boughey, ${ }^{\dagger}$ Richard A. Whiter, ${ }^{\dagger}$ \\ Suman-Lata Sahonta, ${ }^{\dagger}$ and Sohini Kar-Narayan ${ }^{*}{ }^{\dagger}$ \\ ${ }^{\dagger}$ Department of Materials Science and Metallurgy, University of Cambridge, 27 Charles Babbage Road, Cambridge CB3 0FS, United \\ Kingdom \\ ${ }^{\ddagger}$ Instituto de Ciencia de Materiales de Sevilla, C.S.I.C.-Universidad de Sevilla, C. Américo Vespucio n ${ }^{\circ}$, 41092 Sevilla, Spain
}

\section{Supporting Information}

ABSTRACT: A flexible and robust piezoelectric nanogenerator (NG) based on a polymer-ceramic nanocomposite structure has been successfully fabricated via a cost-effective and scalable templateassisted hydrothermal synthesis method. Vertically aligned arrays of dense and uniform zinc oxide $(\mathrm{ZnO})$ nanowires (NWs) with high aspect ratio (diameter $\sim 250 \mathrm{~nm}$, length $\sim 12 \mu \mathrm{m}$ ) were grown within nanoporous polycarbonate (PC) templates. The energy conversion efficiency was found to be $\sim 4.2 \%$, which is comparable to previously reported values for $\mathrm{ZnO}$ NWs. The resulting NG is found to have excellent fatigue performance, being relatively immune to detrimental environmental factors and mechanical failure, as the constituent $\mathrm{ZnO}$ NWs remain embedded and protected inside the polymer matrix.
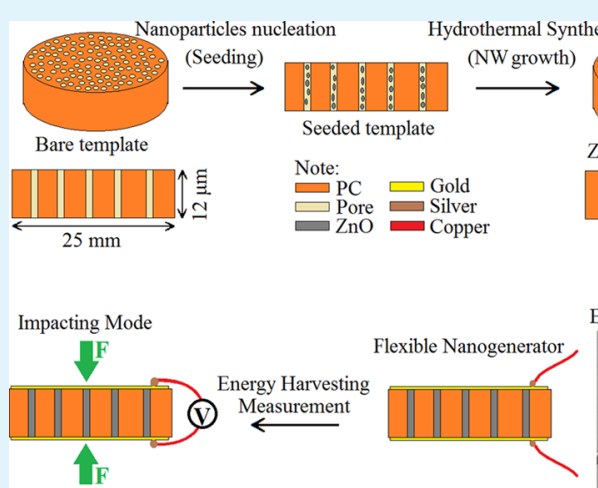

ydrothermal Synthesis (NW growth) $\stackrel{\text { Nowth) }}{\longrightarrow}$
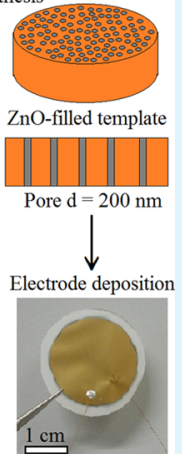

KEYWORDS: ZnO nanowires, hydrothermal synthesis, piezoelectric effect, nanogenerators, energy harvesting

$\mathrm{H}$ arvesting energy from ambient sources in our environment has drawn considerable attention in light of the demand for alternative energy solutions for "small power" electronic applications that currently mostly rely on traditional power sources such as batteries. Ubiquitous and easily accessible ambient vibrations could provide a convenient means to power devices such as wireless sensors, wearable and portable electronics, and even biomedical implants. ${ }^{1,2}$ Energy harvesting (EH) devices based on piezoelectric nanogenerators (NGs) are well-suited for scavenging power directly from small-scale ambient vibrations such as body movement, heartbeats, wind and fluid flow, by directly converting mechanical energy to electrical energy. Piezoelectric NWs, in particular, are typically more sensitive to lowamplitude vibrations than their bulk or thin-film counterparts, allowing for their integration into small-scale and efficient NG devices. $^{3-5}$ Piezoelectric ceramic materials typically possess relatively high piezoelectric coefficients, ${ }^{6}$ but these materials are prone to mechanical failure because they simultaneously possess high stiffness constants. ${ }^{3}$ From a vibrational EH point of view, the brittle nature of piezoelectric ceramics renders them a less than ideal materials choice for NGs, whereas piezoelectric polymers can offer greater flexibility at the expense of output voltage for the same amount of strain. ${ }^{3}$ We therefore aim to fabricate polymer-ceramic nanocomposites as NG devices, based on well-aligned piezoelectric $\mathrm{ZnO} \mathrm{NWs}$ that are embedded and supported in a flexible polymer matrix. The low-temperature template-assisted hydrothermal synthesis method developed in this work is easily scalable ${ }^{7,8}$ and results in flexible NGs with as-grown $\mathrm{ZnO} \mathrm{NWs}$ that require minimal postdeposition processing and exhibit stable $\mathrm{EH}$ performance over time. We demonstrate the $\mathrm{EH}$ performance of a NG fabricated in this method by subjecting it to low-level periodic vibrations and recording the output voltage and current characteristics, and optimizing the output power across an impedance-matched load resistance. Importantly, the structure of our NG renders the $\mathrm{ZnO}$ NWs well-protected from environmental factors, including light, that are known to be detrimental to $\mathrm{EH}$ performance. ${ }^{9-11}$

$\mathrm{ZnO}$ NWs have been previously synthesized by various techniques, but among them, wet chemical methods ${ }^{7,12-14}$ such as sol-gel synthesis, ${ }^{15,16}$ electrochemical deposition, ${ }^{15,17}$ and hydrothermal synthesis ${ }^{7,13,14,18-21}$ are more attractive as the growth occurs at a relatively low temperature and thus can be easily scaled up. ${ }^{7}$ There have been many investigations into the growth of $\mathrm{ZnO} \mathrm{NWs}$ onto different substrates, such as indium tin oxide (ITO) coated glass, ${ }^{22,23}$ poly(ethylene terephthalate $)^{24}$ and silicon wafer substrates, ${ }^{18}$ but hydrothermal synthesis was not adopted before to grow solid $\mathrm{ZnO} \mathrm{NWs}$ directly within flexible porous polymer templates. Stassi et al. ${ }^{25}$ reported the synthesis of hollow $\mathrm{ZnO}$ nanotubes (NTs) within PC membranes by aqueous chemical method where the

Received: April 5, 2016

Accepted: May 13, 2016

Published: May 13, 2016 
reaction was conducted at atmospheric pressure within a beaker. The resulting NTs with wall thickness of the order of $10 \mathrm{~nm}$ had low mechanical stability as evidenced by their disintegration upon dissolution of the PC template. In this work, we have developed a novel method to grow high-quality solid $\mathrm{ZnO}$ NWs within flexible PC templates, resulting in particularly high structural integrity of the NWs so that they remain vertically aligned and intact even upon removal of the PC template. To the best of our knowledge, this is the first report on the growth of solid $\mathrm{ZnO} \mathrm{NWs}$ within nanoporous polymer templates using a simple, scalable and low-temperature $\left(<100{ }^{\circ} \mathrm{C}\right)$ hydrothermal synthesis method that can be used to directly fabricate flexible NGs based on the ZnO-PC nanocomposite structure. The NWs obtained by this method are well-aligned, highly ordered and mechanically supported, with the pores of the PC template offering good control over the length and diameter of NWs. Although previously reported polymer $\mathrm{ZnO} \mathrm{NWs}$ embedded in a polymer matrix ${ }^{12}$ were realized using multiple fabrication steps, our template-assisted approach results in $\mathrm{ZnO} \mathrm{NWs}$ embedded in a PC matrix fabricated from a single growth process, with minimal postprocessing steps required for the fabrication of a $\mathrm{ZnO}$ PC nanocomposite NG.

For the hydrothermal synthesis of $\mathrm{ZnO} \mathrm{NWs}$, zinc nitrate and hexamethylenetetramine (HMTA) are the most widely used precursor solution. ${ }^{18}$ The chemical reaction is given by

$$
\mathrm{Zn}^{2+}+2 \mathrm{OH}^{-} \leftrightarrow \mathrm{Zn}(\mathrm{OH})_{2} \rightarrow \mathrm{ZnO}+\mathrm{H}_{2} \mathrm{O}
$$

Here, zinc nitrate is the source of $\mathrm{Zn}^{2+}$ ions while HMTA steadily provides $\mathrm{OH}^{-}$for $\mathrm{ZnO}$ crystal growth. Traditionally, the so-called high-growth-rate aqueous solution ${ }^{26}$ is often used to grow well-aligned $\mathrm{ZnO} \mathrm{NWs}$ onto rigid substrates, which contains not only the precursor solution, but also polyethylenimine (PEI) and ammonium hydroxide that are required to effectively suppress the homogeneous nucleation in the bulk solution, facilitating the heterogeneous nucleation on the substrate and thereby allowing the growth of aligned $\mathrm{ZnO}$ NWs rather than formation of bulk particles. ${ }^{21,23}$ However, the diameter and length of $\mathrm{ZnO}$ NWs obtained largely decreased, and various defects were induced by PEI and ammonium additions, leading to a relatively lower piezoelectric performance. $^{21}$ In this work, we avoided the inclusion of PEI and ammonium in our hydrothermal growth process by instead using the $\mathrm{PC}$ template pores (with average diameter $\sim 227 \pm 92$ $\mathrm{nm}$ and length $\sim 12 \mu \mathrm{m}$ ) to confine the growth of $\mathrm{ZnO}$ NWs, as demonstrated in Figure 1. The figure shows the field-emission scanning electron microscopy (FE-SEM; FEI Nova NanoSEM) images of the PC template before (Figure 1a) and after (Figure 1b) the hydrothermal synthesis of $\mathrm{ZnO} \mathrm{NWs}$, with energy dispersive X-ray mapping (EDX; Bruker XFlash 6100) in Figure 1c. These images confirm a large fraction of the PC pores were filled with $\mathrm{ZnO}$, and the resulting distribution of $\mathrm{ZnO} \mathrm{NW}$ diameter (average $256 \pm 51 \mathrm{~nm}$ ) is shown in Figure $1 \mathrm{~d}$. The NW-filled template can be partially dissolved using chlorobenzene (see Supporting Information part S1) to partly expose the $\mathrm{ZnO}$ NWs thus revealing the $\mathrm{ZnO}-\mathrm{PC}$ nanocomposite structure, as shown in Figure S1a.

The growth method detailed in Supporting Information part S1, comprised of two steps: (I) seeding of the PC template pores in the precursor solution, and (II) subsequent NW growth within a Teflon-sealed stainless steel autoclave reactor. As the NW growth process can be controlled by adjusting reaction parameters e.g. precursor concentration, growth
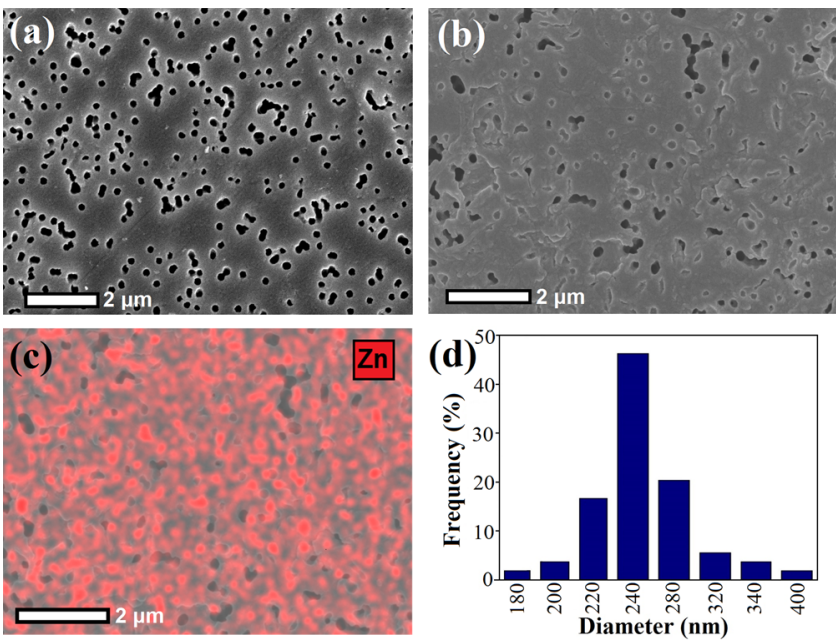

Figure 1. Top-down scanning electron micrographs of undissolved polycarbonate (PC) templates (a) before and (b) after hydrothermal synthesis at $95{ }^{\circ} \mathrm{C}$ for $5 \mathrm{~h}$ with solution refreshing treatment; (c) energy-dispersive $\mathrm{X}$-ray mapping analysis of the $\mathrm{ZnO}$-filled $\mathrm{PC}$ template in $\mathrm{b}$; (d) diameter distribution of $\mathrm{ZnO}$ nanowires (NWs) freed by completely burning the PC template away at $500{ }^{\circ} \mathrm{C}$.

temperature and growth time, it was found that the optimum conditions for the growth of $\mathrm{ZnO}$ NWs within PC templates is $95{ }^{\circ} \mathrm{C}$ for $5 \mathrm{~h}$. To obtain higher density and longer NWs, the precursor solution was "refreshed" twice by repeating the growth steps on the same PC template, with care being taken to remove $\mathrm{ZnO}$ crystals deposited on the surface of the PC template by gently washing in hydrochloric acid (see Supporting Information part S1). To visualize the structure and morphology of the $\mathrm{ZnO}$ nanowires freed from the PC template, the template was burned away at $500{ }^{\circ} \mathrm{C}$ for $0.5 \mathrm{~h}$ at a slow heating rate of $1{ }^{\circ} \mathrm{C}$ per min. Figure 2 shows SEM images of free-standing $\mathrm{ZnO}$ NWs that resulted upon complete removal of the PC template, revealing a dense array of
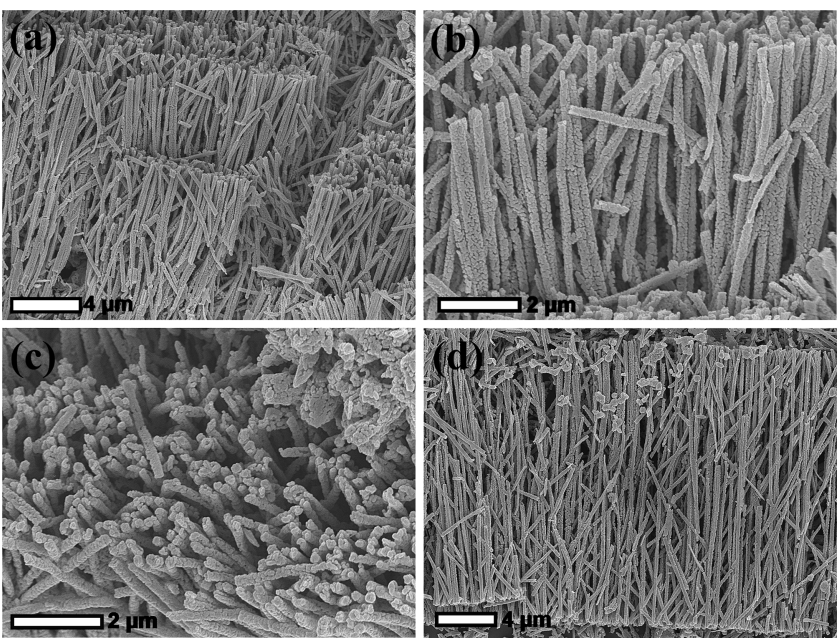

Figure 2. (a) Scanning electron micrographs (SEM) of well-aligned zinc oxide $(\mathrm{ZnO})$ nanowires (NWs) that were freed by completely burning the polycarbonate (PC) template away at $500{ }^{\circ} \mathrm{C}$; (b) enlarged view of free-standing NWs in a; (c) tilted-view of freestanding NWs in a; (d) cross-sectional SEM image of the ZnO-filled PC template that was burned away, leaving only well-aligned $\mathrm{ZnO}$ NWs. 

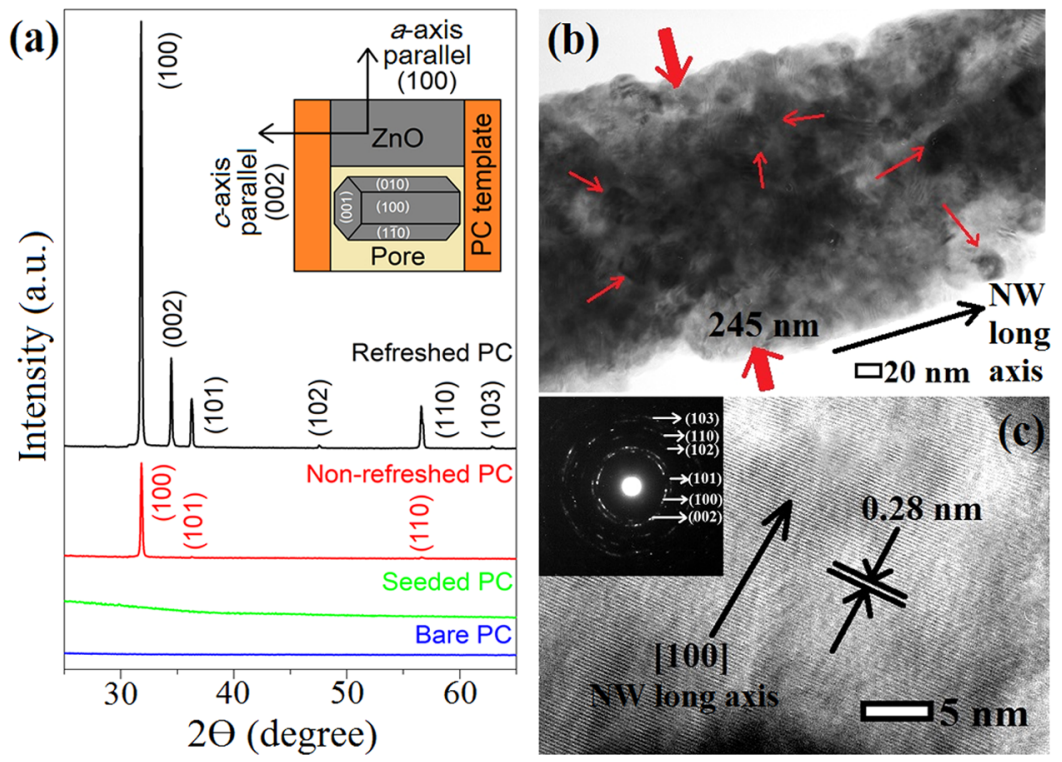

Figure 3. (a) X-ray diffraction patterns of a bare PC template and three PC templates under different growth treatments (seeded, nonrefreshed, and refreshed). The inset demonstrates the growth mechanism of well-aligned polycrystalline ZnO NWs within PC templates; (b) high-resolution transmission electron microscopy (HRTEM) image of a $\mathrm{ZnO}$ NW; (c) enlarged-view of HRTEM image in b, with inset showing the selected area electron diffraction (SAED) pattern with polycrystalline rings of the major crystalline planes indexed.

vertically aligned $\mathrm{ZnO} \mathrm{NWs}$ of uniform length grown within the confinement of the PC template pores. It was also found that the solution-refreshing treatment largely increased the length and density of NWs as more pores were filled (Figure S2f). Although our template removal method allows for easy collection and imaging of the NWs, some NWs were found to partially disintegrate because of the resulting thermal shock and the mechanical stress produced because of the deformation of the PC templates during the burning treatment. The average length of the free-standing NWs was found to be $8.4 \pm 2.1 \mu \mathrm{m}$ which is close to the nominal thickness of the PC template. The $\mathrm{ZnO} \mathrm{NWs}$ grown within PC templates exhibited much higher aspect ratio compared with the NWs grown from the same precursor solution directly onto ITO-coated substrates, where NW lengths were found to be typically less than $4 \mu \mathrm{m}$ (Figure S3).

The orientation and crystalline structure of the templategrown $\mathrm{ZnO} \mathrm{NWs}$ were confirmed using $\mathrm{X}$-ray diffraction studies (XRD; Bruker D8 advanced). The XRD patterns (Figure 3a) from a bare PC template as well as three separate PC templates subjected to different growth treatments (seeded, nonrefreshed and refreshed) are shown. It can be seen that there is no signal detected from the seeded PC template, indicating the seeding process only resulted in noncrystalline $\mathrm{ZnO}$ nuclei rather than NWs. Only the PC templates (refreshed and nonrefreshed) filled with $\mathrm{ZnO}$ NWs show the formation of a dominant peak from (100). The presence of higher order peaks suggest that these NWs may be polycrystalline. The refreshed $\mathrm{ZnO}-\mathrm{PC}$ sample was found to exhibit a more intense signal than the nonrefreshed $\mathrm{ZnO}$-PC sample, indicating that a higher density of NWs was obtained via the solution-refreshing treatment. This is due to the fact that as the $\mathrm{Zn}^{2+}$ source is gradually depleted from the precursor solution, it is difficult to form long NWs in a single growth step, and thus refreshing the precursor solution is an effective approach to obtain longer $\mathrm{NWs}^{12,27}$ under the confinement effect of PC templates. Note that that higher-order peaks are not evident in the XRD pattern of the nonrefreshed sample because of the much weaker signal arising from incompletely formed NWs in the nonrefreshed sample, as compared to the higher density of fully grown NWs in the refreshed sample.

High-resolution transmission electron microscopy (HRTEM; JEOL 4000EXII) images are shown in Figure 3b, c. As indicated from the HRTEM image, the polycrystalline $\mathrm{ZnO} \mathrm{NWs}$ constitute well-formed crystals with sizes ranging between 22 and $30 \mathrm{~nm}$ (shown by arrows in Figure $3 \mathrm{~b}$ ). The average diameter of the $\mathrm{ZnO}$ NWs as calculated from the TEM images is $240 \mathrm{~nm} \pm 40 \mathrm{~nm}$. A lattice fringe spacing of $0.28 \mathrm{~nm}$ reveals the dominant (100) plane growth in the $\mathrm{ZnO}$ NWs along their long-axis. Furthermore, the selected area electron diffraction (SAED) pattern (Figure $3 \mathrm{c}$ inset) characteristically shows the highly crystalline ring pattern, indicative of polycrystalline $\mathrm{ZnO}$.

Compared with the above results, $\mathrm{XRD}$ patterns from $\mathrm{ZnO}$ films and bulk crystals grown hydrothermally from the same precursor solution exhibit no preferred orientation (Figure S4a). Furthermore, the ZnO NWs grown within PC templates exhibit an oriented $a$-axis growth along (100) (illustrated in the inset of Figure 3a) as compared to the $\mathrm{ZnO} \mathrm{NWs}$ grown directly onto ITO-coated substrates which preferentially grow along a (002) axis (Figure S4b). This indicates the fabricated $\mathrm{ZnO}$ NWs grown within PC templates show preferential $c$-axis orientation perpendicular to the pore walls as a result of being confined by the template pores, ${ }^{25}$ and not simply due to the hydrothermal growth process. The NWs are thus formed of single-crystalline nanoparticles with the $c$-axis parallel to the plane of the template stacked on top of one another within each pore, with smaller crystallites of random orientations present as well, possibly arising due to interface imperfections. The shorter NWs grown onto ITO-coated substrates are likely to be single crystalline with very strong alignment exhibited in XRD patterns (Figures S3 and S4b), while longer $\mathrm{ZnO}$ NWs grown within $\mathrm{PC}$ templates show polycrystallinity due to fusing of the nanoparticles or nanorods together, corresponding to higher order peaks. The observed alignment of $\mathrm{ZnO} \mathrm{NWs}$ is crucial for the NG applications as further electrical poling treatment is not required to align the piezoelectric domains. ${ }^{28}$ 

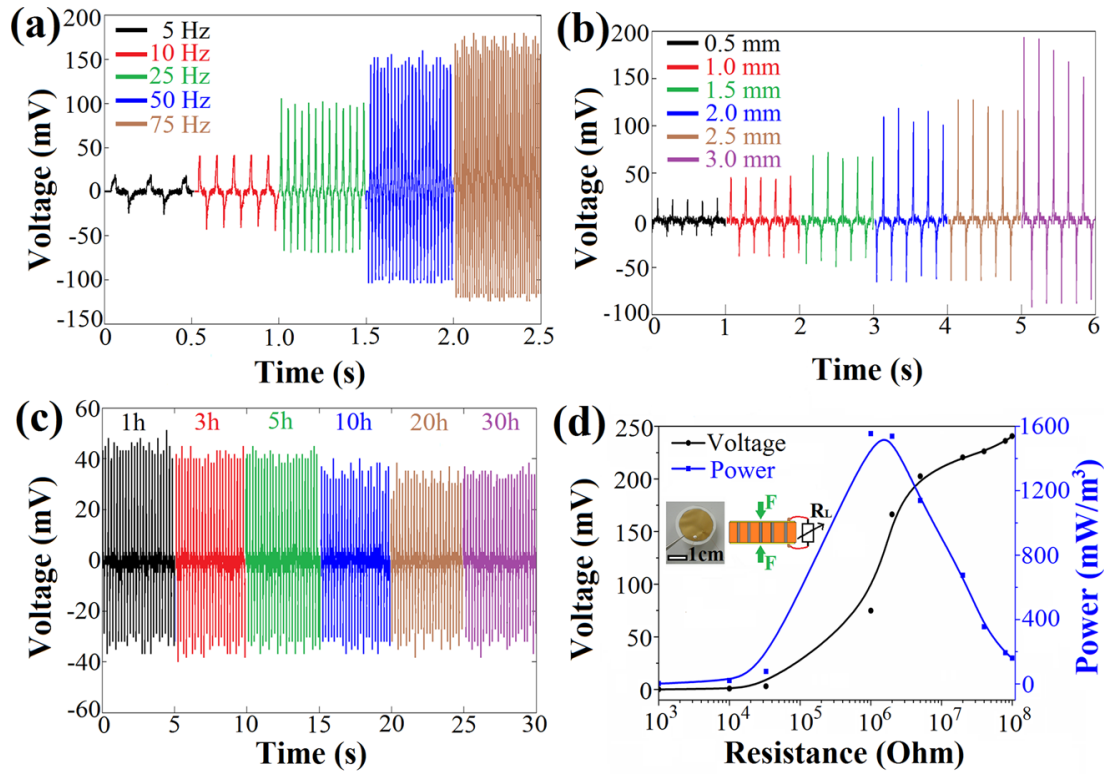

Figure 4. Open circuit voltage output of a flexible ZnO-PC based NG under the application of a periodic impacting force (a) at a fixed amplitude of $\sim 0.5 \mathrm{~mm}$ with various frequencies ranging from 5 to $75 \mathrm{~Hz}$, and (b) at a fixed frequency of $5 \mathrm{~Hz}$ with various amplitudes ranging from 0.5 to $3 \mathrm{~mm}$. (c) Fatigue test of a $\mathrm{ZnO}-\mathrm{PC}$ based NG, continually impacting at a frequency of $5 \mathrm{~Hz}$ and amplitude of $\sim 1 \mathrm{~mm}$ on the same NG for $30 \mathrm{~h}$ ( 540000 impacting cycles in total), and the data after $1 \mathrm{~h}$ (18k cycles), $3 \mathrm{~h}$ (54k cycles), $5 \mathrm{~h}$ (90k cycles), $10 \mathrm{~h}$ (180k cycles), $20 \mathrm{~h}(360 \mathrm{k}$ cycles), and $30 \mathrm{~h}$ (540k cycles) were recorded accordingly. (d) Piezoelectric output voltage and power generated by a ZnO-PC based NG across different load resistors $R_{\mathrm{L}}$, under the application of a periodic impacting force at a frequency of $75 \mathrm{~Hz}$ and amplitude of $\sim 1 \mathrm{~mm}$, in which the inset shows a photo of an actual NG device and a schematic illustrating the direction of impacting.

Finally, flexible, lightweight, and inexpensive NGs were fabricated by sputtering gold electrodes of thickness $\sim 30 \mathrm{~nm}$ onto the surfaces of the $\mathrm{ZnO} \mathrm{NW}$-filled PC template (refreshed sample), with $\mathrm{Cu}$ wires attached using $\mathrm{Ag}$ paint for electrical access. The piezoelectric response of these flexible NGs were measured by using a vibrational energy harvesting system previously described by Whiter et al. $^{8}$ to record the output voltage and power under different loading conditions, in response to periodic mechanical impacting, as shown in Figure 4. Our NG was subjected to uniaxial compression in order to take advantage of the vertical alignment of the $\mathrm{ZnO}$ NWs with $a$-axis orientation along their lengths. The fabricated NGs were tested at frequencies ranging from 5 to $75 \mathrm{~Hz}$, and different amplitudes of the oscillating arm ranging from 0.5 to $3 \mathrm{~mm}$, while keeping the other parameter fixed (see Supporting Information part S3 for details of the energy harvesting measurement setup). The observed open-circuit voltage output $V_{\text {OC }}$ exhibited positive peaks corresponding to compression of the NGs, whereas the negative peaks arise due to the releasing of strain. ${ }^{22}$ The peak-to-peak value of $V_{\mathrm{OC}}$ increased with both increasing frequency (Figure $4 \mathrm{a}$ ) as well as amplitude (Figure $4 \mathrm{~b})$. On the basis of these frequency- and amplitude-dependent tests, a significant increase of $V_{\mathrm{OC}}$ could be obtained through impacting forces of higher frequency and/or amplitude. Electrical output characteristics for an impacting periodic force of frequency $5 \mathrm{~Hz}$ and amplitude $1 \mathrm{~mm}$, akin to gentle finger tapping (Figure S6), are presented in Figure S9 for comparison. We have also measured the output voltage in response to simple bending by hand, as shown in Figure S7. A blank PC template was tested under the same impacting conditions as the $\mathrm{ZnO}-\mathrm{PC} \mathrm{NGs}$ to confirm that the measured electrical output was solely due to the piezoelectric $\mathrm{ZnO}$ NWs (Figure S8a). Also, it was found that compared to the nonrefreshed samples (Figure S8b), the solution-refreshed samples could generate up to an order of magnitude higher output voltage as well as current, which was consistent with the observation of longer and higher-density NWs being formed.

To highlight the mechanical stability and robustness of our $\mathrm{NG}$, we conduct rigorous fatigue testing of our device by subjecting it to up to 540000 continuous impacting cycles, i.e., $30 \mathrm{~h}$ of continuous testing at $5 \mathrm{~Hz}$, as shown in Figure 4c. We find that after an initial $\sim 10 \%$ drop in the peak value of $V_{\mathrm{OC}}$ occurring after $10 \mathrm{~h}$ of testing, the output remains stable for up to $30 \mathrm{~h}$ of testing. Furthermore, our NG was tested over a period of 4 months with negligible degradation in performance. Our results are particularly significant given that such extensive fatigue data is rarely presented in the literature for $\mathrm{ZnO} N W$ based NGs (see Table S1).

To evaluate how much electrical power the ZnO-PC NGs can generate in a practical application, we connected the NG to different load resistors $R_{\mathrm{L}}(1 \mathrm{k} \Omega$ to $100 \mathrm{M} \Omega)$ to determine the maximum power output that can be achieved through impedance matching. ${ }^{29}$ The output power was found to peak at a resistance of $\sim 1 \mathrm{M} \Omega$ with a value of power density $\sim 1600$ $\mathrm{mW} / \mathrm{m}^{3}$ (Figure $4 \mathrm{~d}$ ), for an effective NG area of $\sim 3 \mathrm{~cm}^{2}$. Note that only $12 \%$ of this area comprises the piezo-active $\mathrm{ZnO}$ NWs, as this fraction corresponds to the porosity of the PC template. The output power can be greatly enhanced by stacking several devices in parallel and connecting them electrically in series, ${ }^{8}$ or by subjecting the NG to a higher impacting force (Figure S6a). It is generally difficult to quantitatively compare electrical output of NGs due to the large differences in geometry and mechanical excitations reported $^{29,30}$ (see Table S1). However, from the measured electrical output characteristics, the energy conversion efficiency $^{29-31}$ of our NG was evaluated to be $\sim 4.2 \%$ (see Supporting Information part S5), which is comparable to previously reported $\mathrm{ZnO} \mathrm{NWs} .^{30}$ 
In summary, we have successfully developed a templateassisted hydrothermal synthesis method to grow dense and well-aligned $\mathrm{ZnO} \mathrm{NWs}$ with uniform diameter and length within PC templates that provide flexibility and robustness to the resulting NGs. Because of the confined growth process, all fabricated $\mathrm{ZnO} \mathrm{NWs}$ demonstrate strong alignment along (100) preferred orientation, which is beneficial to their piezoelectric performance under uniaxial compression. While higher piezoelectric performance is expected of $c$-axis oriented NWs, this is outweighed by the benefit of prolonged and robust piezoelectric performance as seen in the superior fatigue performance of our NG. Furthermore, the compliant and flexible PC template allows for effective straining of the $\mathrm{ZnO}$ NWs upon application of stress, and can be easily mounted on curved surfaces without causing the NG to fracture. A single NG of area $3 \mathrm{~cm}^{2}$ and thickness $\sim 12 \mu \mathrm{m}$ generates a peak output power density of $\sim 1600 \mathrm{~mW} / \mathrm{m}^{3}$ across a load resistance of $\sim 1 \mathrm{M} \Omega$, and excellent fatigue performance with an energy conversion efficiency that is typically higher than $\mathrm{ZnO}$ thin films or bulk samples. The as-grown $\mathrm{ZnO} \mathrm{NWs}$ are well-protected from detrimental environmental factors and mechanical failure within the PC template, and can be easily incorporated into NGs without the need to be removed from the template. The solution-based fabrication method is low-cost and easily scalable and can thus be used to produce cheap and stable NGs for EH applications.

\section{ASSOCIATED CONTENT}

\section{S Supporting Information}

The Supporting Information is available free of charge on the ACS Publications website at DOI: 10.1021/acsami.6b04041.

(S1) Experimental section for the fabrication of wellaligned $\mathrm{ZnO} \mathrm{NWs}$ grown within PC templates; (S2) growth mechanism of well-aligned $\mathrm{ZnO}$ NWs within PC templates; (S3) fabrication and measurement techniques of flexible ZnO-PC based NGs; (S4) supplementary piezoelectric energy harvesting measurements on flexible ZnO-PC-based NGs; (S5) conversion efficiency calculation of flexible $\mathrm{ZnO}$-PC-based $\mathrm{NGs}$ (PDF)

\section{AUTHOR INFORMATION}

\section{Corresponding Author}

*E-mail: sk568@cam.ac.uk.

\section{Author Contributions}

C.O fabricated the samples and performed most of the measurements reported. S.K $-\mathrm{N}$ and P.S.J designed and guided the experimental work. A.D and S-L.S conducted the high resolution TEM imaging. R.A.W and F.L.B built the energy harvesting measurement setup. C.O and S.K-N cowrote the paper. All authors discussed the results and commented on the paper.

\section{Funding}

This work was financially supported by a grant from the European Research Council through an ERC Starting Grant (Grant no. ERC-2014-STG-639526, NANOGEN).

\section{Notes}

The authors declare no competing financial interest.

\section{ACKNOWLEDGMENTS}

The authors thank Yeonsik Choi for discussions and experimental support. S.K.-N., C.O., and A.D. are grateful for financial support from the European Research Council through an ERC Starting Grant (Grant no. ERC-2014-STG-639526, NANOGEN). F.L.B. and R.A.W. thank the EPSRC Cambridge NanoDTC, EP/G037221/1, for studentship funding. P.S.J. acknowledges the support of TEP-1900 and Talentia Postdoc Program, cofunded by the European Union's Seventh Framework Program, Marie Skłodowska-Curie actions (COFUND Grant Agreement 267226) and the Ministry of Economy, Innovation, Science and Employment of the Junta de Andalucia. S-L.S acknowledges support through the EPSRC grant EP/M010589/1. Supporting data for this paper is available at the DSpace@Cambridge data repository (https:// www.repository.cam.ac.uk/handle/1810/256007).

\section{ABBREVIATIONS}

$\mathrm{NG}$, nanogenerator

$\mathrm{ZnO}$, zinc oxide

NWs, nanowires

PC, polycarbonate

$\mathrm{EH}$, energy harvesting

NGs, nanogenerators

ITO, indium tin oxide

NTs, nanotubes

HMTA, hexamethylenetetramine

PEI, polyethylenimine

SEM, scanning electron microscopy

EDX, energy dispersive X-ray

NW, nanowire

XRD, X-ray diffraction

HRTEM, high-resolution transmission electron microscopy

\section{REFERENCES}

(1) Briscoe, J.; Dunn, S. Piezoelectric Nanogenerators-A Review of Nanostructured Piezoelectric Energy Harvesters. Nano Energy 2015, 14, 15-29.

(2) Wang, X. Piezoelectric Nanogenerators-Harvesting Ambient Mechanical Energy at the Nanometer Scale. Nano Energy 2012, 1, 1324.

(3) Crossley, S.; Whiter, R. A.; Kar-Narayan, S. Polymer-Based Nanopiezoelectric Generators for Energy Harvesting Applications. Mater. Sci. Technol. 2014, 30, 1613-1624.

(4) Espinosa, H. D.; Bernal, R. A.; Minary-Jolandan, M. A Review of Mechanical and Electromechanical Properties of Piezoelectric Nanowires. Adv. Mater. 2012, 24, 4656-4675.

(5) Wang, Z. L.; Song, J. Piezoelectric Nanogenerators Based on Zinc Oxide Nanowire Arrays. Science 2006, 312, 242-246.

(6) Bowen, C. R.; Kim, H. A.; Weaver, P. M.; Dunn, S. Piezoelectric and Ferroelectric Materials and Structures for Energy Harvesting Applications. Energy Environ. Sci. 2014, 7, 25-44.

(7) $\mathrm{Xu}$, S.; Wang, Z. L. One-Dimensional ZnO Nanostructures: Solution Growth and Functional Properties. Nano Res. 2011, 4, 10131098.

(8) Whiter, R. A.; Narayan, V.; Kar-Narayan, S. A Scalable Nanogenerator Based on Self-Poled Piezoelectric Polymer Nanowires with High Energy Conversion Efficiency. Adv. Energy Mater. 2014, 4, 1400519-1400525.

(9) Jalali, N.; Briscoe, J.; Tan, Y. Z.; Woolliams, P.; Stewart, M.; Weaver, P. M.; Cain, M. G.; Dunn, S. ZnO Nanorod Surface Modification with PDDA/PSS Bi-Layer Assembly for Performance Improvement of $\mathrm{ZnO}$ Piezoelectric Energy Harvesting Devices. J. SolGel Sci. Technol. 2015, 73, 544-549.

(10) Jalali, N.; Woolliams, P.; Stewart, M.; Weaver, P. M.; Cain, M. G.; Dunn, S.; Briscoe, J. Improved Performance of $\mathrm{P}-\mathrm{n}$ JunctionBased $\mathrm{ZnO}$ Nanogenerators through CuSCN-Passivation of $\mathrm{ZnO}$ Nanorods. J. Mater. Chem. A 2014, 2, 10945-10951. 
(11) Hu, Y.; Lin, L.; Zhang, Y.; Wang, Z. L. Replacing a Battery by a Nanogenerator with 20 v Output. Adv. Mater. 2012, 24, 110-114.

(12) Xu, S.; Qin, Y.; Xu, C.; Wei, Y.; Yang, R.; Wang, Z. L. SelfPowered Nanowire Devices. Nat. Nanotechnol. 2010, 5, 366-373.

(13) Cao, H. L.; Qian, X. F.; Gong, Q.; Du, W. M.; Ma, X. D.; Zhu,

Z. K. Shape and Size Controlled Synthesis of Nanometre $\mathrm{ZnO}$ from a Simple Solution Route at Room Temperature. Nanotechnology 2006, 17, 3632-3636.

(14) Cheng, B.; Samulski, E. T. Hydrothermal Synthesis of OneDimensional $\mathrm{ZnO}$ Nanostructures with Different Aspect Ratios. Chem. Commun. 2004, 986.

(15) Ottone, C.; Bejtka, K.; Chiodoni, A.; Farías, V.; Roppolo, I.; Canavese, G.; Stassi, S.; Cauda, V. Comprehensive Study of the Templating Effect on the $\mathrm{ZnO}$ Nanostructure Formation within Porous Hard Membranes. New J. Chem. 2014, 38, 2058-2065.

(16) Zhou, H.; Wong, S. S. A Facile and Mild Synthesis of 1-D ZnO, $\mathrm{CuO}$, and a-Fe2O3 Nanostructures and Nanostructured Arrays. ACS Nano 2008, 2, 944-958.

(17) Zhang, Y.; Ram, M. K.; Stefanakos, E. K.; Goswami, D. Y. Synthesis, Characterization, and Applications of $\mathrm{ZnO}$ Nanowires. J. Nanomater. 2012, 2012, 1-22.

(18) Vayssieres, L. Growth of Arrayed Nanorods and Nanowires of $\mathrm{ZnO}$ from Aqueous Solutions. Adv. Mater. 2003, 15, 464-466.

(19) Tian, J. H.; Hu, J.; Li, S. S.; Zhang, F.; Liu, J.; Shi, J.; Li, X.; Tian, Z.-Q.; Chen, Y. Improved Seedless Hydrothermal Synthesis of Dense and Ultralong ZnO Nanowires. Nanotechnology 2011, 22, 245601245609.

(20) Liu, B.; Zeng, H. C. Hydrothermal Synthesis of ZnO Nanorods in the Diameter Regime of 50 Nm. J. Am. Chem. Soc. 2003, 125, 44304431.

(21) Chen, L. Y.; Yin, Y. T.; Chen, C.; Chiou, J. W. Influence of Polyethyleneimine and Ammonium on the Growth of $\mathrm{ZnO}$ Nanowires by Hydrothermal Method. J. Phys. Chem. C 2011, 115, 20913-20919.

(22) Rivera, V. F.; Auras, F.; Motto, P.; Stassi, S.; Canavese, G.; Celasco, E.; Bein, T.; Onida, B.; Cauda, V. Length-Dependent Charge Generation from Vertical Arrays of High-Aspect-Ratio ZnO Nanowires. Chem. - Eur. J. 2013, 19, 14665-14674.

(23) Podrezova, L. V.; Cauda, V.; Stassi, S.; Cicero, G.; Abdullin, K. a.; Alpysbaeva, B. E. Properties of $\mathrm{ZnO}$ Nanorods Grown by Hydrothermal Synthesis on Conductive Layers. Cryst. Res. Technol. 2014, 49, 599-605.

(24) Unalan, H. E.; Hiralal, P.; Rupesinghe, N.; Dalal, S.; Milne, W. I.; Amaratunga, G. a J. Rapid Synthesis of Aligned Zinc Oxide Nanowires. Nanotechnology 2008, 19, 255608-2556012.

(25) Stassi, S.; Cauda, V.; Ottone, C.; Chiodoni, A.; Pirri, C. F.; Canavese, G. Flexible Piezoelectric Energy Nanogenerator Based on $\mathrm{ZnO}$ Nanotubes Hosted in a Polycarbonate Membrane. Nano Energy 2015, 13, 474-481.

(26) Xu, C.; Shin, P.; Cao, L.; Gao, D. Preferential Growth of Long

$\mathrm{ZnO}$ Nanowire Array and Its Application in Dye-Sensitized Solar Cells. J. Phys. Chem. C 2010, 114, 125-129.

(27) Han, Z.; Li, S.; Li, J.; Chu, J.; Chen, Y. Facile Synthesis of ZnO Nanowires on FTO Glass for Dye-Sensitized Solar Cells. J. Semicond. 2013, 34, 074002-074006.

(28) Xu, S.; Hansen, B. J.; Wang, Z. L. Piezoelectric-NanowireEnabled Power Source for Driving Wireless Microelectronics. Nat. Commun. 2010, 1, 93-97.

(29) Crossley, S.; Kar-Narayan, S. Energy Harvesting Performance of Piezoelectric Ceramic and Polymer Nanowires. Nanotechnology 2015, 26, 344001-344009.

(30) $\mathrm{Wu}, \mathrm{W}$. High-performance piezoelectric nanogeneratores for self-powered nanosystems: quantitative standards and figures of merit. Nanotechnology 2016, 27, 112503-112508.

(31) Briscoe, J.; Jalali, N.; Woolliams, P.; Stewart, M.; Weaver, P. M.; Cain, M.; Dunn, S. Measurement Techniques for Piezoelectric Nanogenerators. Energy Environ. Sci. 2013, 6, 3035-3045. 